\title{
Efectos de la diabetes en el embarazo sobre el recién nacido
}

\author{
Darío Chavarro; Olga Lucía Melo; Juan Guillermo Bastidas; Paola D’Aleman; Paola Devis; Sonia Gonzales; \\ Daniel Rojas; Humberto Reynales*
}

\begin{abstract}
RESUMEN: La presencia de diabetes en el embarazo afecta a un número significativo de mujeres y es considerada como un factor con importante papel en la morbilidad y mortalidad perinatal. Con el objetivo de determinar los efectos de esta enfermedad sobre el recién nacido, se realizó un estudio retrospectivo en 35 casos de mujeres con diabetes mellitus o diabetes gestacional que habían tenido un parto previamente. Se escogieron además al azar, 26 controles de partos de mujeres normales para establecer las comparaciones respectivas. Se observó un efecto de la enfermedad en variables como el Apgar, la talla y los perímetros cefálico y torácico de los recién nacidos. No se encontraron efectos sobre el peso como tampoco diferencias sobre la presencia de malformaciones. Por la importancia de esta situación tanto para la madre como para el recién nacido, se recomiendan medidas de control estricto durante el embarazo de estas mujeres, así como en la atención del recién nacido buscando minimizar los riesgos.
\end{abstract}

PALABRAS CLAVES: Diabetes mellitus, diabetes gestacional, resultado perinatal, apgar.

SUMMARY: Diabetes in pregnant women is an important factor affecting perinatal morbidity and mortality. Oriented to determine the effects of the diabetes in the new born, a retrospective study was performed. 35 cases of women including diabetes mellitus or gestational diabetes were reviewed. Additionaly 26 normal women were selected ramdomly to compare the variables of interest. In some variables as Apgar test, height, cephalic and thoracic perimeters in the new born were affected. No alterations in weight neither congenital malformations were detected as result of the diabetes, comparing the two groups. Because of the importance of this disease for the mother and for the new born, and special management is required looking for minimizing the risks.

KEY WORDS: Diabetes mellitus, gestational diabetes, perinatal outcome.

\section{Introducción}

La Diabetes Mellitus es una enfermedad que afecta entre 1 a $2 \%$ de la población, produciendo deletéreos efectos en diversos órganos, mientras que en el embarazo se considera que se presenta en un 0.2 a $0.3 \%$ como Diabetes preexistente y de 1 a $5 \%$ como Diabetes Gestacional (1-4). La enfermedad ocasiona efectos sobre el feto que incluyen anomalías de diversa índole, además de las de tipo congénito en un $10 \%$ de los casos. En las madres la mortalidad asociada con diabetes es del $12 \%$ en los casos en que la enfermedad se presenta con anterioridad al embarazo; y en un 7\% cuando se clasifica como Diabetes Gestacional (5-6).

En el recién nacido, el test de APGAR se considera como un instrumento de gran valor para determinar el estado del recién nacido, así como su pronóstico principalmente desde el punto de vista neurológico. Sobre este aspecto hay estudios que sugieren un mayor riesgo de secuelas neurológicas en hijos de madres diabéticas (713), mientras que otro concluye que no existe diferencia significativa en el desarrollo neurológico entre los hijos de madres diabéticas y no diabéticas (14).

\footnotetext{
* Facultad de Medicina. Universidad del Rosario. Hospital de San José. Santa Fe de Bogotá, Colombia.
}

Se busca con este estudio determinar los efectos de la diabetes en el embarazo sobre diferentes variables del recién nacido como son el Apgar, su peso, talla y perímetros; como también sobre la madre y la evolución de su gestación.

\section{Materiales y métodos}

El estudio se realizó en el Hospital de San José, un Centro Universitario privado, de tercer nivel, ubicado en una zona deprimida de la ciudad de Santa Fe de Bogotá, con una utilización de servicios dada aproximadamente por un $65 \%$ de pacientes provenientes del Instituto de Seguros Sociales, un $15 \%$ de empresas, un $10 \%$ de pacientes particulares y un $10 \%$ de Asistencia Social.

Se revisaron 35 historias clínicas del Departamento de Gineco-Obstetricia entre mayo de 1982 y noviembre de 1992, de mujeres en embarazo que tuvieran el diagnóstico de diabetes. Simultáneamente y al azar se tomaron 26 historias clínicas de pacientes normales que presentaron su embarazo en fechas similares a las de aquellas diabéticas. Este último se utilizó como grupo de control para establecer las diferencias entre las variables consideradas.

Las variables a estudiar además de la presencia o no de la enfermedad, fueron principalmente peso, talla, perímetros cefálico y torácico del recién nacido, así como el Apgar al minuto y a los cinco minutos. Además 
se analizó la importancia de la edad y estado civil de la madre, la presencia de hipertensión arterial, la edad y la fórmula gestacional, el control prenatal y las complicaciones presentadas en el embarazo y en el parto.

Los criterios de inclusión establecidos fueron: para las pacientes con diagnóstico de diabetes, éstas podrían ser tipo I o tipo II insulino dependientes, no insulino dependientes o insulino requirientes. Además aquellas con diagnóstico de diabetes gestacional durante el embarazo en estudio. Para las pacientes control se tuvieron en cuenta mujeres embarazadas, sin diagnóstico de diabetes, y con una fecha de parto similar a aquella de las pacientes caso. Ninguna otra variable fue considerada como criterio de inclusión. Se excluyeron aquellas pacientes cuyas historias clínicas no presentaran la información requerida de forma adecuada.

La información fue procesada mediante el programa Epi-Info. Se establecieron frecuencias relativas y absolutas de cada variable y para cada uno de los grupos. Se obtuvieron además promedios de las variables continuas. El análisis se hizo para el grupo de pacientes con diagnóstico de Diabetes Mellitus de aparición previa al em- barazo (DM), para el grupo con diagnóstico de Diabetes Gestacional (DG), y para un tercer grupo que integraba los dos anteriores (DT); todos comparados con el de pacientes normales $(\mathrm{N})$. Se realizaron comparaciones de promedios y proporciones con la prueba $t$, y se obtuvieron valores de $\mathrm{p}$ e intervalos de confianza (IC) considerando una significancia del $95 \%$.

\section{Resultados}

Se incluyeron un total de 35 mujeres embarazadas con diagnóstico de diabetes, de las cuales 11 tenían la enfermedad previa al embarazo, y 24 tenían diabetes gestacional. Además se tomaron 26 controles de pacientes normales. Se encontró una diferencia significativa en el promedio de edad de los tres grupos, siendo menor el de las pacientes normales (DM p =0.013, DG p =0.00003, DT $p=0.098)$. En cuanto al estado civil se encontró una diferencia significativa entre las de unión libre de los grupos N y DT $(\mathrm{p}=0.04)$ y entre $\mathrm{N}$ y DG ( $\mathrm{p}=0.042)$. Sin embargo al determinar el riesgo (OR) comparando las casadas con las de unión libre, este no fue estadísticamente significativo (Tabla 1).

\section{Tabla 1} CARACTERISTICAS GENERALES DE LA POBLACION EN ESTUDIO
HOSPITAL DE SAN JOSE, SANTA FE DE BOGOTA

\begin{tabular}{|c|c|c|c|c|c|}
\hline & & $\begin{array}{l}\text { Diabetes } \\
\text { Mellitus }\end{array}$ & $\begin{array}{r}\text { Diabetes } \\
\text { Gestacional }\end{array}$ & $\begin{array}{r}\text { Total } \\
\text { Diabetes }\end{array}$ & Normal** \\
\hline Edad & $\begin{array}{r}\text { Rango } \\
\text { Promedio } \\
\text { Total } \\
\text { Valor P }\end{array}$ & $\begin{array}{r}22-39 \\
30.8 \\
11 \\
0.013^{*}\end{array}$ & $\begin{array}{r}22-40 \\
32.6 \\
34 \\
0.00003^{*}\end{array}$ & $\begin{array}{r}22-40 \\
32.1 \\
25 \\
0.0098^{*}\end{array}$ & $\begin{array}{r}17-26 \\
25.2 \\
26\end{array}$ \\
\hline $\begin{array}{l}\text { Estado civil } \\
\text { Casada }\end{array}$ & $\begin{array}{r}\# \\
\% \\
\text { Valor } \mathrm{P}\end{array}$ & $\begin{array}{r}6 \\
54.5 \\
0.49\end{array}$ & $\begin{array}{r}16 \\
66.7 \\
0.08\end{array}$ & $\begin{array}{r}22 \\
62.9 \\
0.11\end{array}$ & $\begin{array}{r}11 \\
42.3\end{array}$ \\
\hline Soltera & $\begin{array}{l}\# \\
\%\end{array}$ & 0 & $\begin{array}{r}1 \\
4.2\end{array}$ & $\begin{array}{r}1 \\
2.9\end{array}$ & 0 \\
\hline U. Libre & $\begin{array}{r}\# \\
\% \\
\text { Valor } \mathrm{P}\end{array}$ & $\begin{array}{r}4 \\
36.4 \\
0.23\end{array}$ & $\begin{array}{r}7 \\
29.2 \\
0.042^{*}\end{array}$ & $\begin{array}{r}11 \\
31.4 \\
0.04 *\end{array}$ & $\begin{array}{r}15 \\
57.7\end{array}$ \\
\hline Separada & $\begin{array}{l}\# \\
\%\end{array}$ & $\begin{array}{r}1 \\
9.1\end{array}$ & 0 & $\begin{array}{r}1 \\
2.9\end{array}$ & 0 \\
\hline $\begin{array}{l}\text { Casada } \\
\text { vs U. Libre }\end{array}$ & $\begin{array}{r}\text { OR } \\
\text { IC }\end{array}$ & $\begin{array}{r}2.05 \\
0.37-12.20\end{array}$ & $\begin{array}{r}3.12 \\
0.83-12.16\end{array}$ & $\begin{array}{r}2.73 \\
0.83-9.13\end{array}$ & \\
\hline
\end{tabular}

* Estadísticamente significativo

** Grupo tomado como base de comparación

$\mathrm{OR}=$ Odds Ratio o Desigualdad Relativa

$\mathrm{IC}=$ Intervalo de confianza $95 \%$ 
Sobre la presencia de hipertensión arterial como patología asociada, no se observaron diferencias entre los grupos, aunque si se observó una tendencia cuando se comparó la tensión arterial diastólica de los grupos $\mathrm{N}$ y DG, $(\mathrm{p}=0.047)$, siendo menor en este último. Tampoco se observó diferencia en cuanto a la presencia de complicaciones durante el embarazo o en el momento del parto (Tabla 2).

La fórmula gestacional de las pacientes mostró que es mayor el número de madres con Diabetes Gestacional que tiene tres embarazos, comparadas con las normales, mientras que en estas últimas es más común tener solo un embarazo. Se encuentra una diferencia significativa con relación al hecho de no haber presentado abortos previos, siendo esto mayor en las pacientes normales que en el grupo DM $(\mathrm{p}=0.013)$ con respecto al grupo DT, existe tendencia a la diferencia $(\mathrm{p}=0-05)$. Sinembargo aunque los valores de riesgo (OR) para la ocurrencia de abortos son llamativos, éstos no son estadísticamente significativos (Tabla 3).

Como era de esperarse, el uso de medicamentos durante el embarazo es mayor entre las diabéticas, especialmente en el grupo con Diabetes Mellitus, que entre las normales. Mientras que no hay diferencias en cuanto a la asistencia al control prenatal si se presentan respecto a la finalización del embarazo, mediante la práctica de la operación cesárea, la cual es mayor en el grupo de DG $(\mathrm{OR}=3.78 \mathrm{IC}=1.01-14.6)$ que en las pacientes normales (Tabla 3).

El resultado del parto no muestra diferencias en cuanto al sexo del recién nacido, como tampoco de la edad gestacional alcanzada al momento del parto. Entre

Tabla 2

PRESENCIA DE HTA Y COMPLICACIONES DE LA POBLACION EN ESTUDIO

\begin{tabular}{|c|c|c|c|c|c|}
\hline & & $\begin{array}{l}\text { Diabetes } \\
\text { Mellitus }\end{array}$ & $\begin{array}{r}\text { Diabetes } \\
\text { Gestacional }\end{array}$ & $\begin{array}{r}\text { Total } \\
\text { Diabetes }\end{array}$ & Normal** \\
\hline \multicolumn{6}{|l|}{ HTA } \\
\hline \multirow[t]{2}{*}{$\mathrm{Si}$} & $\#$ & 3 & 5 & 8 & 4 \\
\hline & $\%$ & 27.3 & 20.8 & 22.9 & 15.4 \\
\hline \multirow[t]{5}{*}{ No } & \# & 8 & 19 & 27 & 22 \\
\hline & $\%$ & 72.7 & 79.2 & 77.1 & 84.6 \\
\hline & Valor $\mathrm{P}$ & 0.61 & 0.80 & 0.46 & \\
\hline & OR & 2.06 & 1.45 & 1.63 & \\
\hline & IC & $0.24-15.12$ & $0.27-8.36$ & $0.37-8.34$ & \\
\hline \multirow[t]{2}{*}{ TA Sistólica } & Promedio & 125.5 & 119.6 & 121.5 & 119.6 \\
\hline & Valor P & 0.25 & 1 & 0.58 & \\
\hline \multirow[t]{2}{*}{ TA Diastólica } & Promedio & 73.6 & 68.3 & 70 & 75.7 \\
\hline & Valor P & 0.65 & $0.047 *$ & 0.10 & \\
\hline \multicolumn{6}{|l|}{ Complicaciones } \\
\hline \multirow[t]{5}{*}{ Embarazo } & \# & 2 & 8 & 10 & 9 \\
\hline & $\%$ & 18.2 & 33.3 & 28.6 & 34.6 \\
\hline & Valor $\mathbf{P}$ & 0.40 & 0.92 & 0.61 & \\
\hline & OR & 0.42 & 0.94 & 0.76 & \\
\hline & IC & $0.04-2.78$ & $0.25-3.56$ & $0.22-2.58$ & \\
\hline \multirow[t]{5}{*}{ Parto } & $\#$ & 0 & 2 & 2 & 1 \\
\hline & $\%$ & & 8.3 & 5.7 & 3.8 \\
\hline & Valor $\mathrm{P}$ & 1 & 0.81 & 0.78 & \\
\hline & OR & $* * *$ & 2.27 & 1.52 & \\
\hline & IC & & $0.11-139.3$ & $0.07-92.9$ & \\
\hline
\end{tabular}

* Estadísticamente significativo

** Grupo tomado como base de comparación

*** Indeterminado

$\mathrm{OR}=$ Odds Ratio o Desigualdad Relativa

$\mathrm{IC}=$ Intervalo de confianza $95 \%$ 
Tabla 3

RESULTADO DE CARACTERISTICAS DEL RECIEN NACIDO EN LOS DIFERENTES GRUPOS DE ESTUDIO

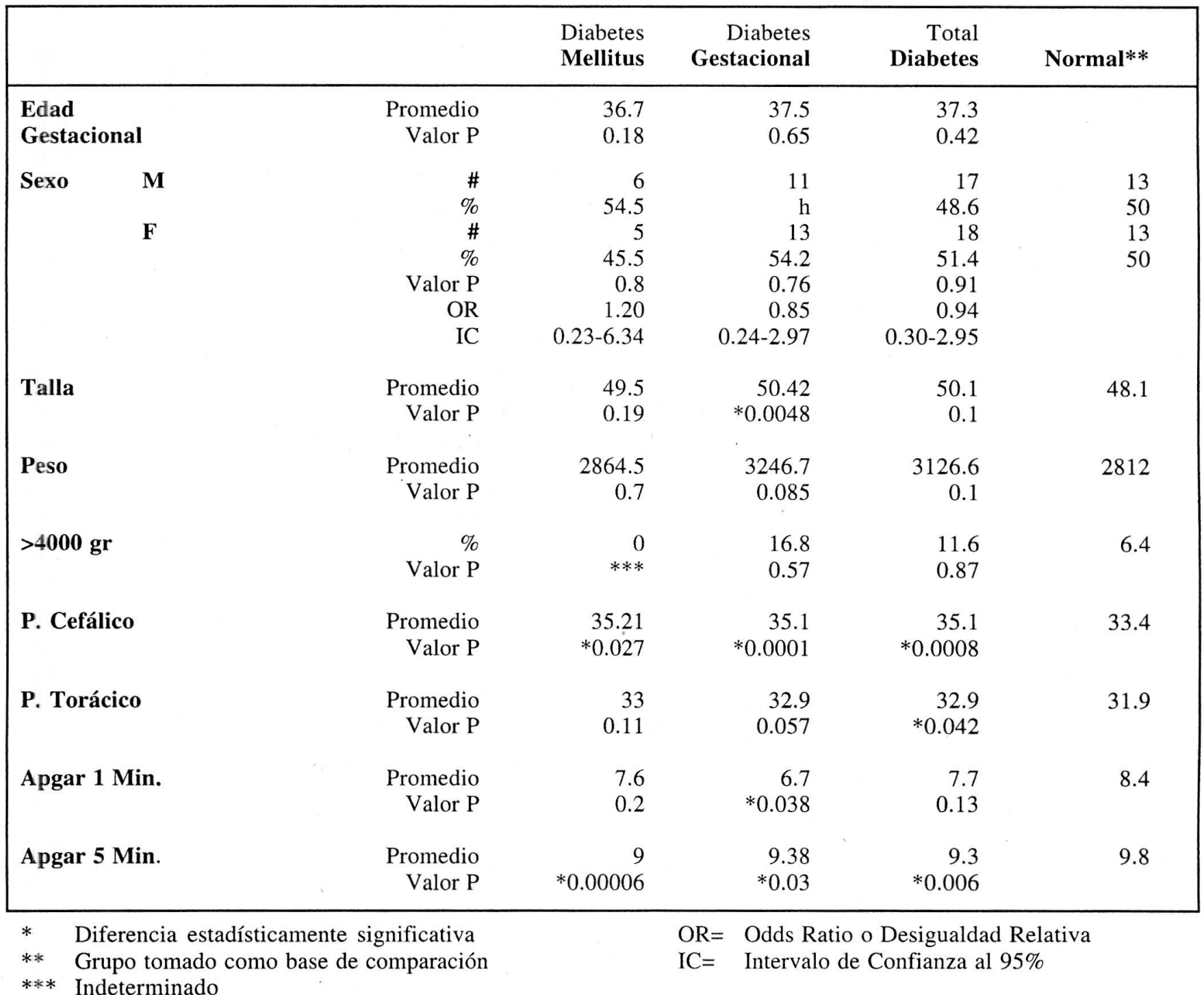

las otras variables se encuentra que la talla del recién nacido es significativamente mayor en las pacientes con diabetes gestacional que en las normales $(p=0.0048)$, mientras que en el peso del recién nacido no se observan diferencias entre los grupos. Aunque el porcentaje de recién nacidos con peso mayor de 4.000 gramos es mayor en el grupo de diabetes gestacional y en el de diabetes total que en las normales, esta diferencia no es significativa. En cuanto a los perímetros, en el cefálico existen diferencias altamente significativas entre los grupos, siendo menor el de DG ( $\mathrm{p}=0.00096)$, DT $(\mathrm{p}=0.00077)$ y $\mathrm{DM}(\mathrm{p}=0.027)$. En el perímetro torácico la diferencia es menos marcada pero es también significativa en el grupo DT. El Apgar al minuto es menor en el grupo DG que en el grupo $\mathrm{N}(\mathrm{p}=0.038)$, mientras que a los cinco minutos es altamente significativa la diferencia entre los grupos, siendo mayor en el grupo N (Tabla 4).

\section{Discusión}

Se observa como la relación entre Diabetes Gestacional y Diabetes Mellitus en los casos estudiados es de 2 a 1 , lo cual ratifica la importancia de realizar pruebas de tamizaje en la mujer embarazada, orientados a descartar esta enfermedad de aparición durante la gestación, sin descuidar el seguimiento de aquellas pacientes que llegan a un embarazo con un diagnóstico claro de Diabetes Mellitus.

Llama la atención la comparación por edad de las pacientes, donde aquellas con la enfermedad tienen entre 30 y 32 años, en contraste con las pacientes normales con un promedio de edad de 25 años. Esto es un criterio que también debe ser considerado tanto en el tamizaje como en la consejería previa al embarazo de esas pacientes. Los hallazgos respecto al estado civil parecieran ser accidenta- 
Tabla 4

FORMULA GESTACIONAL, USO DE MEDICAMENTOS Y PRACTICA DE CESAREA EN LOS DIFERENTES GRUPOS DE ESTUDIO

\begin{tabular}{|c|c|c|c|c|c|}
\hline & & $\begin{array}{l}\text { Diabetes } \\
\text { Mellitus }\end{array}$ & $\begin{array}{r}\text { Diabetes } \\
\text { Gestacional }\end{array}$ & $\begin{array}{r}\text { Total } \\
\text { Diabetes }\end{array}$ & Normal** \\
\hline \multicolumn{6}{|l|}{ Embarazos } \\
\hline \multirow[t]{3}{*}{1} & \# & 2 & 4 & 6 & 12 \\
\hline & $\%$ & 18.2 & 16.7 & 17.1 & 46.2 \\
\hline & Valor P & 0.14 & $* 0.025$ & $* 0.014$ & \\
\hline \multirow[t]{3}{*}{2} & \# & 2 & 4 & 6 & 7 \\
\hline & $\%$ & 18.2 & 16.7 & 17.1 & 26.9 \\
\hline & Valor P & 0.68 & 0.38 & 0.35 & \\
\hline \multirow[t]{3}{*}{3} & \# & 1 & 9 & 10 & 3 \\
\hline & $\%$ & 9.1 & 37.5 & 28.6 & 11.5 \\
\hline & Valor $\mathrm{P}$ & 0.72 & $* 0.031$ & 0.1 & \\
\hline \multirow[t]{3}{*}{4} & $\#$ & 3 & 5 & 8 & 3 \\
\hline & $\%$ & 27.3 & 20.8 & 22.9 & 11.5 \\
\hline & Valor P & 0.42 & 0.55 & 0.32 & \\
\hline \multicolumn{6}{|l|}{ Abortos } \\
\hline \multirow{3}{*}{ Rovitus } & \# & 3 & 5 & 8 & 4 \\
\hline & $\%$ & 27.3 & 20.8 & 22.9 & 15.4 \\
\hline & Valor P & 0.61 & 0.8 & 0.46 & \\
\hline \multirow[t]{5}{*}{ No } & \# & 4 & 16 & 20 & 21 \\
\hline & $\%$ & 36.4 & 66.7 & 57.1 & 80.8 \\
\hline & Valor P & $* 0.013$ & 0.25 & 0.051 & \\
\hline & OR & 3.94 & 1.64 & 2.10 & \\
\hline & IC & $0.39-34.27$ & $0.3-9.6$ & $0.46-10.9$ & \\
\hline \multicolumn{6}{|c|}{ Uso de Medicamentos } \\
\hline \multicolumn{6}{|c|}{ 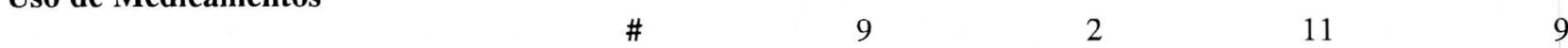 } \\
\hline & $\%$ & 81.8 & 8.3 & 31.4 & 34.6 \\
\hline & Valor $\mathrm{P}$ & $* 0.0086$ & $* 0.025$ & 0.79 & \\
\hline & OR & 8.50 & 0.17 & 0.87 & \\
\hline & IC & $1.27-91.56$ & $0.02-1.02$ & $0.26-2.91$ & \\
\hline \multirow[t]{5}{*}{ Parto por Cesárea } & \# & 5 & 16 & 21 & 9 \\
\hline & $\%$ & 45.5 & 66.7 & 60 & 34.6 \\
\hline & Valor P & 0.62 & $* 0.023$ & $* 0.049$ & \\
\hline & OR & 1.57 & 3.78 & 2.83 & \\
\hline & IC & $0.29-8.23$ & $1.01-14.6$ & $0.88-9.37$ & \\
\hline
\end{tabular}

* Diferencia estadísticamente significativa

** Grupo tomado como base de comparación
$\mathrm{OR}=$ Odds Ratio o Desigualdad Relativa

$\mathrm{IC}=$ Intervalo de confianza les sin importancia epidemiológica, sinembargo cada vez más se cuestiona el papel de esta variable interpretada como el soporte emocional/familiar de las pacientes y el desarrollo de complicaciones en el embarazo.

Acerca de la fórmula gestacional de los grupos de estudio, resultó ser más frecuente la primigravidez entre los controles normales, mientras que las diabéticas tienen un mayor número de embarazos, lo cual se ha demostrado previamente en algunos estudios, pero de la misma manera ha sido rebatido en otros. Respecto a la presencia de abortos previos, esta situación es más frecuente entre las diabéticas, específicamente aquellas con Diabetes Mellitus. Acerca de la presencia de alteraciones en la presión arterial otros estudios tampoco han encontrado diferencias significativas entre los grupos (15-16).

Curiosamente no se observan diferencias respecto al hecho de asistencia a control prenatal. Aunque no se evaluaron aspectos relacionados con la frecuencia o 
calidad del control realizado a cada uno de los grupos. Este ha sido considerado como un elemento que favorece el pronóstico del embarazo y del parto en todo tipo de pacientes en gestación.

Las complicaciones que son bien reconocidas en el embarazo y parto en este grupo de pacientes, no presentaron diferencias en este estudio. Sobre la forma de terminación del embarazo, fue mayor por cesárea entre las pacientes diabéticas que en las normales, aunque algunos consideran que si no existe macrosomía, el parto vaginal está indicado, como sería en este caso. Al respecto algunos estudios reportan bajas frecuencias de cesáreas en estas pacientes, 10 al $20 \%$, mientras que otros hablan de cifras del 50 al $90 \%(6,8,17-18)$.

Sobre la edad gestacional, de acuerdo con la literatura no es necesario tratar de disminuirla en los casos de pacientes diabéticas, lo cual es corroborado con los resultados obtenidos, donde no se observan diferencias entre los grupos (17-18).

De las variables del recién nacido en las cuales se observaron algunos cambios están la talla, siendo mayor entre las pacientes diabéticas. Aunque se podría esperar que se correlacionara con la macrosomía fetal, al comparar los promedios de peso de los recién nacidos no se encontraron diferencias entre los grupos. Aunque un mayor porcentaje de hijos de diabéticas pesaron más de 4.000 gramos, esta diferencia no fue significativa, pero si son menores que los reportados por otros autores. Por otra parte la macrosomía definida como peso mayor de 4.500 gramos sólo se presentó un caso de Diabetes Gestacional. Estos dos hallazgos coinciden con la literatura (5).

Los perímetros cefálico y torácico resultaron ser menores entre los hijos de madres diabéticas. $\mathrm{Al}$ respecto, estudios de seguimiento de recién nacidos hijos de diabéticas con perímetros cefálicos inferiores, han mostrado un menor desarrollo intelectual (14). Respecto al Apgar, mostró una disminución significativa entre los hijos de las madres diabéticas comparado con las normales, lo cual se observa de manera más marcada en la medición de los 5 minutos. Esto ha sido anunciado previamente, junto con otros factores que pueden causar la disminución del Apgar. También es claro que se pueden obtener valores normales en el test si se logra un control adecuado de la glicemia en la embarazada. Finalmente, a pesar de estar bien establecida la presencia de alteraciones del recién nacido incluyendo malformaciones congénitas, éstas no se presentaron en los casos de estudio (19-20).

Las posibles consecuencias de la diabetes ya sea como antecedente o cuando hace su aparición durante la gestación obliga a promover la realización de actividades de tamizaje y prevención en los grupos de alto riesgo. Sabiendo que con medidas adecuadas se pueden obtener resultados similares a aquellos de pacientes no diabéticas, su implementación es totalmente justificada, así como la realización de consejería previa a la concepción sobre los efectos de la enfermedad sobre el feto, el efecto del embarazo sobre la diabetes así como de la diabetes sobre el embarazo $(5-6,14,16-17,21)$.

\section{BIBLIOGRAFIA}

1. Harrison. Principles of Internal Medicine, McGraw-Hill Ed, 13th ed, 1994; 1979-1999.

2. Centers for disease Control, Perinatal mortality and congenital malformations in infants born to women with insulin-dependent diabetes mellitus -United States, Canada and Europe- 1940-1988. JAMA, 1990; 264: 437-441.

3. Deorovari AK., Kabra SK., Paul VK et al. Perinatal outcome of infants born to diabetic mothers. Indian J. Pediatr, 1991; 28: 1271-1275.

4. Hod M., Diamant YZ. The offspring of a diabetic mother short and long range implications. Isr J Med Sci. 1992; 28: 81-86.

5. Cordero L., Landon MB. Infant of the diabetic mother, Clin Perinatol, 1993; 20(3): 635-648.

6. Rivera-Rueda MA., Barranco-Jaubert A et al. Child of insulin dependent diabetic mother: Neonatal impact, Bol Med Hosp Infant Mex. 1993; 50(5): 321-327.

7. Vohr BR. Long term follow up of the infant of the diabetic mother. In: Gabbe SG, OH W Ed. Infant of the diabetic mother: report of the ninety-third Ross conference on pediatric research, Columbus Ohio, Ross Laboratories, 1987; 159-167.

8. Churchill JA., Berendes HW., Nemores J., Neuropsychological deficits in children of diabetic mothers, Am. J. Obstet. Gynecol. 1969; 105: 257-268.

9. Stehbens JA., Baker OL., Kitchell M. Outcome of ages 1, 3 and 5 years of children born to diabetic women, Am. J. Obstet. Gynecol. 1977; 127: 408-413.

10. Yssing M. Long term prognosis of children born to mothers diabetic when pregnant. In Camerini-Davalus RA, Cole HS, Eds. Early diabetics in early life, New York, Academic Press, 1975; 575-586.

11. Haworth JC., McRae KN., Dilling LA. Prognosis of infants of diabetic mothers in relation to neonatal hypoglycemia. Rev. Med. Child. Neurol. 1976; 18: 471-479.
12. Rizzo T., Metzger BE., Burns WJ., Burns K. Correlations between antepartum maternal metabolism and intelligence of offspring. $\mathrm{N}$. Engl. J Med. 1991; 325: 911-916.

13. Rizzo T., Freinkel N., Metzger BE., Hatcher R., Bruns WJ., Barglow P. Correlations between antepartum maternal metabolism and newborn behavior, Am. J. Obstet. Gynecol. 1990; 163: 1458-1464.

14. Sells CJ., Robinson NM., Brown Z., Knopp RH., Long term developmental follow up of infants of diabetic mothers. J. Pediatr. 1994; 125: S9-17.

15. Patterson CC., Carson DJ., Hadden DR., Waugh NR., Cole SK. A case-control investigation of perinatal risk factors for childhood IDDM in Northern Ireland and Scotland, Diabetes Care, 1994; 17(5): 376-381.

16. Demarini S., Mimouni F., TSang RC., Khoury J., Hertzberg V. Impact of metabolic control of diabetes during pregnancy on neonatal hypocalcemia: a randomized study. Obstet. Gynecol. 1994; 83(6): 918-922.

17. Lurie S., Matzkel A., Weissman A., Gotibe Z., Friedman A. Outcome of preganancy in Class $A 1$ and $A 2$ gestational diabetic patients delivered beyond 40 weeks gestation, Am. J. Perinatol. 1992; 9(5-6): 484-488.

18. Robin SH., Cohen MM., Levinton CM., Kelly EN., Farine D. The premature infant: anesthesia for cesarean delivery, Anesth Analg, 1994; 78(5): 912-917.

19. Litwak LE., Mileo-Vaglio R., Fried T., De-Sancho H., Alvarez A., Althabe O., Gutman RA. Intensified insulin therapy in the management of gestational diabetes. Medicina. B. Aires, 1992; 52(6): 523-533.

20. Thompson D., Dansereau J., Creed M., Ridell L. Thight glucose control results in normal perinatal outcome in 150 patients with gestational diabetes. Obstet. Gynecol. 1994; 83: 362-366.

21. Barrs VA. Diabetes and pregnancy, in Medical problems in pregnancy, Med. Clin. of North. Am. 1989; 73(3): 685-697. 